\title{
Femtosecond Laser Post-Processing of Metal Parts Produced by Laser Additive Manufacturing
}

\author{
Ilya Mingareev', Tobias Bonhoff ${ }^{2}$, Ashraf F. El-Sherif ${ }^{3}$, Martin Richardson ${ }^{1}$ \\ ${ }^{1}$ Townes Laser Institute, CREOL, University of Central Florida, 4000 Central Florida Blvd., Orlando, FL, 32816, USA \\ ${ }^{2}$ Fraunhofer Institute for Laser Technology, Steinbachstrasse 15, 52074 Aachen, Germany \\ ${ }^{3}$ Military Technical College, Cairo, Egypt \\ Author e-mail address: mingareev@ucf.edu
}

\begin{abstract}
High-repetition rate femtosecond laser radiation was utilized to improve surface quality of metal parts manufactured by laser additive techniques. This novel approach can be used to postprocess parts made of heat-sensitive materials, and to attain the designed net shape with micrometer precision.
\end{abstract}

\section{Summary}

Laser Additive Manufacturing (LAM) is a cutting-edge manufacturing approach that is based on consecutive application of material layers and the transfer of geometrical information from the digital design data to the resulting near-net shape [1]. Laser Metal Deposition (LMD) and Selective Laser Melting (SLM) are the two well-established LAM techniques that utilize serial powder materials including superalloys, ceramics, and multi-material compounds e.g. wear and thermal protective coatings [2]. Given the near-net shape nature of LAM, a certain amount of mechanical post-processing, i.e. CNC milling, is required to improve the surface finish and the consistency with the design data.

Femtosecond laser machining is an established micro-processing technique that makes advantage of the ultrafast deposition of the optical energy into the material. By using laser pulses with sub-picosecond duration, the heataffected zone at the workpiece can be significantly reduced compared to longer pulses, and various heat-sensitive materials can be processed with sub-micron precision [3].

In this paper we present our study of femtosecond laser post-processing of different components manufactured by SLM and LMD from Ni-base superalloys. Various 3D-shaped metal parts made of IN718 and IN625 superalloys were irradiated with tightly focused femtosecond laser light (Amplitude Satsuma, $\mathrm{t}_{\mathrm{p}}<400 \mathrm{fs}, \lambda=1030 \mathrm{~nm}, \mathrm{f}_{\mathrm{rep}}=0.5-5$ $\mathrm{MHz}$ ). Different scanning approaches were used to increase the ablation efficiency at flat and curved surfaces of the workpiece. Laser pulse energy was varied to optimize the resulting surface finish and geometrical consistency. Surface roughness was determined before and after the processing.

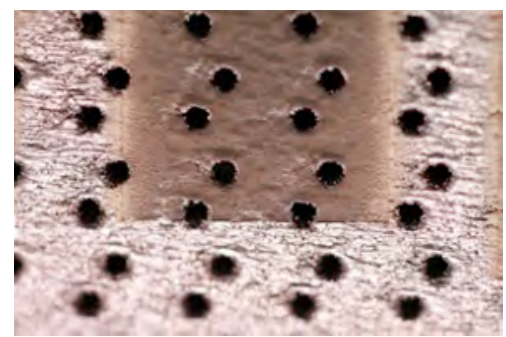

Fig. 1. A $5 \times 5 \mathrm{~mm}^{2}$ processed area on an IN718 superalloy part with curved geometry and holes.

The resulting surface quality was investigated by optical microscopy and white-light interferometry. Compared to the unprocessed regions, the areas irradiated with fs-laser radiation exhibit a reduction in surface roughness from about $100 \mu \mathrm{m}$ to a few microns, depending on the process parameters and surface complexity. The use of highrepetition rate ultrafast laser radiation enables the efficient removal of the excessive material while precisely following the predetermined part geometry.

\section{References}

[1] A. Gasser, G. Backes, I. Kelbassa, A. Weisheit, K. Wissenbach, Laser Additive Manufacturing: Laser Metal Deposition (LMD) and Selective Laser Melting (SLM) in Turbo-Engine Applications, Laser Technik Journal 7(2) 58-63 (2010).

[2] D.D. Gu, W. Meiners, K. Wissenbach, R. Poprawe, Laser additive manufacturing of metallic components: materials, processes and mechanisms. International Materials Reviews, 57(3), 133-164 (2012).

[3] B.N. Chichkov, C. Momma, S. Nolte, F. von Alvensleben, A. Tünnermann, Femtosecond, picosecond and nanosecond laser ablation of solids. Applied Physics A: Materials Science \& Processing, 63(2), 109-115 (1996). 